\title{
ESTIMATIVA DA RADIAÇÃO SOLAR GLOBAL DIÁRIA EM FUNÇÃO DO POTENCIAL DE ENERGIA SOLAR NA SUPERFÍCIE DO SOLO
}

\author{
André Belmont Pereira ${ }^{1 *}$; Augusto Leandro Vrisman ${ }^{2,4}$; Emerson Galvani3 ${ }^{3,5}$ \\ ${ }^{1}$ Depto. de Ciência do Solo e Engenharia Agrícola - UEPG - Campus de Uvaranas, Bloco F. - CEP: 84010-330 - Ponta \\ Grossa, PR. \\ ${ }^{2}$ Graduando em Agronomia - UEPG. \\ ${ }^{3}$ Pós-Graduando em Energia na Agricultura - FCA/UNESP, C.P. 237 - CEP: 18603-970 - Botucatu, SP. \\ ${ }^{4}$ Bolsista CNPq. \\ ${ }^{5}$ Bolsista FAPESP. \\ *Autor correspondente <abelmont@uepg.br>
}

RESUMO: A radiação solar recebida na superfície do solo, além de sua relevância a diversas atividades humanas diárias, desempenha um papel importante no crescimento e desenvolvimento de plantas. Este trabalho teve por objetivo desenvolver e aferir um modelo de estimativa da densidade de fluxo de radiação solar global, em função do potencial de energia solar à superfície, no município de Ponta Grossa, PR, Brasil (latitude $25^{\circ} 13^{\prime} \mathrm{S}$, longitude $50^{\circ} 03^{\prime} \mathrm{W}$, altitude $880 \mathrm{~m}$ ). Os valores estimados do potencial de energia solar obtidos em função de apenas uma medida realizada na passagem meridiana do sol foram confrontados com os valores medidos por um actinógrafo de placa bimetálica de Robitzsch para aqueles dias que apresentaram razão de insolação superior a 0,85 . Este conjunto de dados foi submetido a uma análise de regressão linear simples, obtendo-se bom ajuste entre valores observados e calculados. Foi utilizado o método baseado no potencial de energia solar à superfície para estimativa dos coeficientes $a$ e $b$ da equação de Angström para a localidade em questão. A metodologia foi eficiente para quantificar a densidade de fluxo de radiação solar global, com rapidez e simplicidade, bem como revelaram que o critério de estimativa do potencial de energia solar gerou valores equivalentes aqueles obtidos pela metodologia clássica de Angström. O conhecimento do potencial de energia solar disponível e da densidade de fluxo de radiação global é de suma importância na estimativa da demanda máxima de evaporação, do consumo hídrico de culturas irrigadas e para o dimensionamento de artifícios de engenharia solar, tais como secadores, aquecedores, fogões solares, refrigeradores etc.

Palavras-chave: irradiância global, densidade de fluxo, modelagem, radiometria solar

\section{ESTIMATION OF DAILY GLOBAL SOLAR RADIATION AS A FUNCTION OF THE SOLAR ENERGY POTENTIAL AT SOIL SURFACE}

\begin{abstract}
The solar radiation received at the surface of the earth, apart from its relevance to several daily human activities, plays an important role in the growth and development of plants. The aim of the current work was to develop and gauge an estimation model for the evaluation of the global solar radiation flux density as a function of the solar energy potential at soil surface. Radiometric data were collected at Ponta Grossa, PR, Brazil (latitude $25^{\circ} 13^{\prime} \mathrm{S}$, longitude $50^{\circ} 03^{\prime} \mathrm{W}$, altitude $880 \mathrm{~m}$ ). Estimated values of solar energy potential obtained as a function of only one measurement taken at solar noon time were confronted with those measured by a Robitzsch bimetalic actinograph, for days that presented insolation ratios higher than 0.85 . This data set was submitted to a simple linear regression analysis, having been obtained a good adjustment between observed and calculated values. For the estimation of the coefficients $a$ and $b$ of Angström's equation, the method based on the solar energy potential at soil surface was used for the site under study. The methodology was efficient to assess the coefficients, aiming at the determination of the global solar radiation flux density, whith quickness and simplicity, having also found out that the criterium for the estimation of the solar energy potential is equivalent to that of the classical methodology of Angström. Knowledge of the available solar energy potential and global solar radiation flux density is of great importance for the estimation of the maximum atmospheric evaporative demand, of water consumption by irrigated crops, and also for building solar engineering equipment, such as driers, heaters, solar ovens, refrigerators, etc.

Key words: global solar irradiance, flux density, modelling, solar radiometry
\end{abstract}

\section{INTRODUÇÃO}

A radiação solar é a fonte primária de todos os fenômenos atmosféricos e de processos físicos, químicos e biológicos observados em ecossistemas agrícolas, podendo ser aproveitada sob várias formas, tais como a captura pela biomassa, o aquecimento de ar e água para fins domésticos e industriais, fotoeletricidade para pequenos potenciais e fontes para ciclos termodinâmicos variados.

Há dois meios básicos nos quais a distribuição geográfica da radiação solar pode ser estudada (Robinson, 1966). O primeiro deles envolve medidas por uma rede de estações meteorológicas relativamente 
próximas umas das outras e o segundo é baseado no uso de fórmulas e constantes físicas. Muitas constantes físicas somente podem ser avaliadas por um número muito pequeno de instituições especializadas, ao passo que medidas radiométricas de densidade de fluxo de radiação solar global são inexistentes em muitas regiões onde são necessárias.

Uma análise dos modelos encontrados na literatura para estimar a radiação solar global demonstra que o uso destes modelos deve ser incentivado e não a disseminação de redes de estações radiométricas (Santos et al., 1983), as quais são nas regiões tropicais de baixa densidade e as séries de medidas radiométricas, quando disponíveis, são geralmente de pouca duração.

Diversos pesquisadores do mundo inteiro têm feito uso da metodologia clássica de Angström para caracterizar o regime de distribuição de energia solar em suas regiões de estudo. Recentemente, Ramachanda \& Subramanian (1997) lançaram mão dessa metodologia para estimar a irradiância solar global com base em um conjunto de dados de 20 anos para Mongalore e de 25 anos para Goa, na Índia. Sahin \& Sen (1998) propuseram um método simples para estimar os coeficientes de Angström, os quais desempenham um papel de importância significativa na relação entre a radiação global e a duração de brilho solar, a partir de dados de 28 estações radiométricas espalhadas pela Turquia, tendo sido as variações dos parâmetros regionais da equação de Angström obtidas para todo o país.

Ampratwum \& Dorvlo (1999) obtiveram várias expressões que incluem as funções de regressão de Angström-Black do tipo linear, logarítmica, linearlogarítmica, quadrática e trigonométrica para estimar a densidade de fluxo de radiação $(\mathrm{Qg})$ a partir do número de horas de brilho solar em uma região árida de Oman. Elagib \& Mansell (2000) investigaram a possibilidade de estabelecer equações específicas mensais para estimar o "input" de energia solar no Sudão, usando dados de 16 estações compreendendo zonas sub-úmidas, semiáridas, áridas e super-úmidas, em função da dependência entre $\mathrm{Qg}$ e outros fatores, tais como latitude, altitude e razão de insolação.

O levantamento do potencial de energia solar para fins de estudos de distribuição geográfica da radiação global no Brasil, irrigação, potencial de produção agrícola e zoneamento agro-climático é feito por redes do Inmet e por instituições de pesquisa com o uso do actinógrafo de placa bimetálica. $\mathrm{O}$ emprego de equipamentos mais precisos, baseados no efeito termoelétrico, bem como de sensores de estações automatizadas é ainda muito incipiente para atender às necessidades de todo território nacional.

Os actinógrafos apresentam limitações quanto à sua precisão, especialmente em dias com elevado grau de nebulosidade, estando condicionados a calibração periódica, a qual nem sempre se procede. No entanto, o processo de avaliação de $\mathrm{Qg}$ baseado no modelo geral de Prescott (1940) e difundido por Penman (1948) ainda vem sendo largamente utilizado em nosso país.

Um método prático e expedito para estimativa da densidade de fluxo de radiação solar global, o qual dispensa a análise de uma exaustiva série de medidas radiométricas e que independe de integrações diárias de actinogramas em dias parcial ou completamente nublados, foi desenvolvido em Botucatu, SP (Pereira \& Villa Nova, 1998), Piracicaba, SP (Villa Nova \& Pereira, 1997), São Paulo, SP (Pereira et al., 1998) e testada em Pelotas, RS (Assis et al., 1998), em função da intensidade máxima de radiação solar observada no instante da passagem meridiana do sol (Villa Nova \& Salati, 1977), apresentando excelentes resultados nessas localidades.

Assim propôs-se, no presente trabalho, o desenvolvimento de um teste comparativo entre o critério de estimativa de $\mathrm{Qg}$ baseado na metodologia clássica de Angström, a partir dos coeficientes a e $\mathbf{b}$ obtidos por Caramori et al. (1985), e aquele fundamentado no monitoramento do potencial máximo de radiação solar no município de Ponta Grossa, PR, Brasil.

\section{MATERIAL E MÉTODOS}

O presente estudo foi desenvolvido utilizando-se dados radiométricos da estação agrometeorológica convencional de Vila Velha, do Instituto Agronômico do Paraná, localizada a uma latitude de $25^{\circ} 13^{\prime} \mathrm{S}$, longitude de $50^{\circ} 03^{\prime} \mathrm{W}$ e altitude de 880 metros.

O clima de Ponta Grossa, PR, classificou-se, segundo o sistema Köppen, como Cfb ou temperado propriamente dito, com verões frescos e sem estação seca definida. A precipitação e a temperatura média anual normais são $1700 \mathrm{~mm}$ e $20^{\circ} \mathrm{C}$, respectivamente.

A densidade de fluxo de radiação solar global foi medida por um actinógrafo de placa bimetálica de Robtizsch e seus totais diários obtidos por integração dos actinogramas, com o uso de um planímetro polar de compensação, Koizumi, tipo KP-27, para os dias completamente limpos, e o número de horas de brilho solar foi obtido através da carbonização das fitas de um heliógrafo Campbell-Stockes.

A intensidade máxima de radiação solar global medida no instante da passagem meridiana do sol $\left(\mathrm{I}_{12}\right)$ foi obtida por cotação dos actinogramas para a série de dias limpos selecionados na localidade estudada.

Para a estimativa da radiação total no topo da atmosfera (Qo), da declinação do sol ( $\delta$ ) e do comprimento do dia ou insolação máxima $(\mathrm{N})$ foram utilizadas as equações adotadas por Pereira \& Villa Nova (1998).

$\mathrm{O}$ raio vetor médio $(\mathrm{r})$, definido pela distância Terra-Sol, expressa em termos de distância média, foi obtido do Anuário Astronômico publicado pelo Instituto Astronômico e Geofísico - IAG - da Universidade de São Paulo, em 1980. 
O potencial de energia solar disponível compreende o máximo possível de radiação solar incidente sobre a superfície do solo, para um determinado dia e local, estando a atmosfera presente e completamente isenta de nuvens durante todo o dia. Seu conhecimento é importante na estimativa da demanda máxima de evaporação, evapotranspiração e em artifícios de engenharia solar, como secadores, aquecedores, fogões solares, refrigeradores, etc.

O modelo genérico adotado para estimar o potencial de energia solar à superfície (Qgmax - $\mathrm{MJm}^{-2}$ $\mathrm{dia}^{-1}$ ) concentrou-se na teoria postulada por Villa Nova \& Salati (1977) e comprovada por Pereira \& Villa Nova (1998), dado por:

$$
\operatorname{Qgmax}=\frac{Q_{o I_{12}}}{\left(\frac{J o}{r^{2}}\right) \cos (\partial-\varphi)}
$$

em que: Qo é a densidade de fluxo de radiação solar incidente sobre uma superfície horizontal colocada no limite mais externo da atmosfera $\left(\mathrm{MJm}^{-2} \mathrm{dia}^{-1}\right) ; l_{12}$ é a intensidade máxima de radiação solar na passagem meridiana do sol $\left(\mathrm{Wm}^{-2}\right) ; J o / r^{2}$ é a constante solar corrigida em função do raio vetor médio $r\left(W^{-2}\right) ; \partial$ e $\varphi$ são a declinação solar e a latitude local, respectivamente (graus).

Para proposição dos fatores de correção a serem introduzidos no modelo apresentado na equação 1 foram utilizados dados radiométricos de um período de 18 meses (setembro de 1987 a janeiro de 1989) e para sua validação na localidade estudada uma outra série independente de dados, compreendendo um período de 24 meses, foi considerada em um estudo de regressão linear (janeiro de 1990 a dezembro de 1991).

De posse do potencial de energia solar à superfície, obtido em função de uma única medida instantânea, os coeficientes empíricos da equação de Angström foram calculados pela metodologia proposta por Pereira \& Villa Nova (1998) para fins de determinação da irradiância global estimada, tendo sido, posteriormente, comparada com os valores observados em actinógrafos. $\mathrm{O}$ mesmo procedimento foi adotado a partir dos coeficientes $\mathbf{a}$ e $\mathbf{b}$ determinados por Caramori et al. (1985), visando-se comparação da performance do modelo proposto com aquela obtida pela metodologia convencional.

No particular a análise estatística aplicada aos dados do presente estudo, paralelamente a obtenção dos valores de coeficientes de correlação $(r)$ e de determinação $\left(\mathbf{r}^{2}\right)$, determinou-se também o índice de concordância d preconizado por Willmott et al. (1985), bem como o índice c proposto por Camargo \& Sentelhas (1995) para indicar a performance dos modelos obtidos para a localidade estudada.
O aquecimento de água por intermédio de coletores solares planos foi determinado, objetivando-se ilustrar o aproveitamento da energia solar em uma de suas várias formas. Para tanto, considerou-se o máximo incremento possível de temperatura da água obtido por metro quadrado de coletor solar, definido pela seguinte expressão:

$$
\Delta T=\frac{\eta \cdot Q g \max . A}{V \cdot d \cdot C p}
$$

em que: $\Delta T$ é o incremento de temperatura da água $\left({ }^{\circ} \mathrm{C}\right.$ dia $\left.^{-1}\right) ; \eta$ é o rendimento dos coletores industriais, assumindo valor médio de 0,3 ; A é a superfície de captura do coletor solar plano $\left(\mathrm{m}^{2}\right)$; $\vee$ é o volume de água considerado $\left(\mathrm{m}^{3}\right)$; d é a densidade da água $\left(\mathrm{kg} \mathrm{m}^{-3}\right) \mathrm{e}$ $\mathrm{Cp}$ refere-se ao calor específico do ar $\left(\mathrm{MJ} \mathrm{kg}^{-10} \mathrm{C}^{-1}\right)$.

\section{RESULTADOS E DISCUSSÃO}

Os valores diários do potencial de energia solar disponível no município de Ponta Grossa, PR, calculados pela metodologia empregada por Pereira \& Villa Nova (1998) e medidos por um actinógrafo de placa bimetálica foram altamente correlacionados, haja visto que a análise estatística aplicada aos dados radiométricos utilizados, mediante estudo de regressão linear simples, revela que mais de $96 \%$ das variações obtidas nas medidas do potencial máximo de energia são explicadas pelos valores estimados pela metodologia em estudo. A equação de regressão linear simples proposta para avaliar o potencial de energia solar diário foi a seguinte:

$$
\begin{aligned}
& \operatorname{Qgmax}_{\text {mad }}=0,9423 \operatorname{Qgmax}_{\text {eft }}\left(\mathrm{MJ} \mathrm{m}^{-2} \mathrm{dia}^{-1}\right) \\
& \mathrm{r}^{2}=0,965 \mathrm{~d}=0,982 \mathrm{n}=142
\end{aligned}
$$

$\mathbf{O}$ valor da estatística $\mathbf{F}$ foi altamente significativo, revelando forte correlação entre os dados medidos e estimados de potencial de energia solar. $\mathrm{O}$ teste $\mathbf{t}$ para o coeficiente linear da equação de regressão para o município de Ponta Grossa, PR, foi significativo ao nível de $1 \%$ de confiabilidade, fato que demonstra que o valor de a é estatisticamente diferente de zero.

O coeficiente de determinação $\left(r^{2}\right)$ e o coeficiente de correlação $(\mathbf{r})$ apenas trazem informações sobre a precisão do modelo matemático obtido, porém nada revelam sobre a sua exatidão. Assim, através da determinação de um índice de concordância proposto por Willmott et al. (1985), observou-se alta exatidão para o modelo de estimativa do potencial de energia como uma função de uma única medida instantânea da densidade de fluxo de irradiância solar feita na passsagem meridiana do sol, a qual foi expressa pelo valor de $\mathbf{d}$ da ordem de 0,982 .

$\mathrm{O}$ índice de desempenho c, dado pelo produto entre $\mathbf{r}$ e d, foi de 0,965 para as condições climáticas da localidade estudada, tendo sido demonstrado ótimo desempenho do modelo obtido, considerando-se o critério de interpretação da performance de modelos proposto por Camargo \& Sentelhas (1985). 
Tanto a precisão, expressa pela dispersão dos dados em torno da reta de tendência, como também a exatidão das estimativas, representada pela flutuação dos dados ao redor da linha de $45^{\circ}$ em um gráfico do tipo 1:1, foram consideravelmente elevadas (Figura 1). Ao contrário do que foi observado por Pereira \& Villa Nova (1998), Villa Nova \& Pereira (1997) e Pereira et al. (1998), o modelo subestima ligeiramente os valores de potencial de energia solar diário para o município de Ponta Grossa, PR. A validação do modelo proposto foi efetuada levando-se em conta um novo conjunto de dados radiométricos independentes (Figura 2). Portanto, a dispersão dos pares ordenados, obtidos pelo confronto entre medidas e estimativas a partir da série radiométrica não considerada na proposição do modelo, é muito pequena, o que se confirma estatisticamente pelos coeficientes de determinação de 0,898 e pelos índices d e c de 0,961 e 0,911, respectivamente. Tal fato reforça a viabilidade de utilização da metodologia proposta no local em estudo.

Embora a hipótese de que o valor instantâneo da radiação direta e difusa transmitidas através de um número $\mathbf{m}$ de massas ópticas da atmosfera, orientado normalmente na direção dos raios solares, seja considerado constante ao longo do dia não seja verdadeira, o modelo constitui excelente aproximação da realidade observada (Figura 3 ), pois nele pode ser admitido que a integração do produto entre a irradiância solar máxima medida na passagem meridiana do sol e o valor do cosseno do ângulo zenital em cada instante, leva à obtenção de uma curva radiométrica estimada muito similar àquela registrada pelos actinogramas em dias completamente limpos.

A Figura 4, elaborada com os dados obtidos por Vrisman (2000), indica que os coeficientes a e b da equação de Angström obtidos a partir da metodologia proposta geraram valores de irradiância global que seguem a tendência dos valores medidos, bem como às estimativas obtidas a partir dos coeficientes calculados por Caramori et al. (1985) pelo uso da metodologia convencional, tendo sido observado um erro sistemático de estimativa de, aproximadamente, 3 MJ m ${ }^{-2} \operatorname{dia}^{-1}$.

A confirmação estatística para o que se menciona a respeito da performance dos modelos de estimativa da densidade de fluxo de radiação (Figura 4) pode ser demonstrada através dos valores de $\mathbf{d}$ e $\mathbf{c}$ equivalentes a 0,827 e 0,752 para a metodologia proposta, e de 0,843 e 0,765 para a metodologia clássica de Angström, respectivamente. A variação nos parâmetros empíricos da regressão obtidos por ambos critérios de estimativa levou à obtenção de valores diários de irradiância global muito próximos, não se evidenciando diferenças que pudessem justificar a preferência de utilização da metodologia convencional em Ponta Grossa, PR, sobre a metodologia proposta por Pereira \& Villa Nova (1998), pois a primeira requer integrações diárias de uma série de medidas muito longa e de difícil cotação em dias parcial e completamente nublados.

A dispersão dos dados apresentada na Figura 4 justifica o emprego da metodologia que dispensa a análise de séries radiométricas extensas na localidade analisada, desde que corrigido o erro sistemático, adotada com o objetivo de avaliar a densidade de fluxo de radiação solar global. Sugere-se que novos estudos sejam conduzidos em diversos locais do Brasil. O uso de dados radiométricas medidos, preferencialmente, por equipamentos baseados no efeito termo-elétrico promoveria a proposição de modelos mais fidedignos em uma dada região ou local, quando se utiliza a metodologia que se propõe, particularmente em municípios onde o monitoramento da irradiância global não constitui medida de rotina. Estudos para melhorar a precisão e a operacionalidade das informações radiométricas em todo território nacional são de suma importância, uma vez que grande parte da pesquisa agrometeorológica é baseada no conhecimento da disponibilidade de energia solar em agro-ecossistemas, sendo, portanto, a fonte primária de todos os processos físicos e biológicos que ocorrem na biosfera.

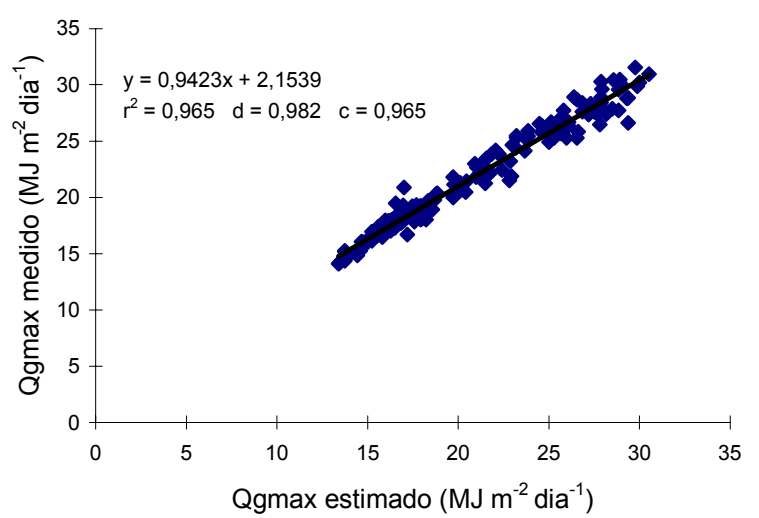

Figura 1 - Relação entre o potencial de energia solar medido pelo actinógrafo de Robitzsch e o potencial estimado pela metodologia proposta por Pereira \& Villa Nova (1998) para Ponta Grossa, PR.

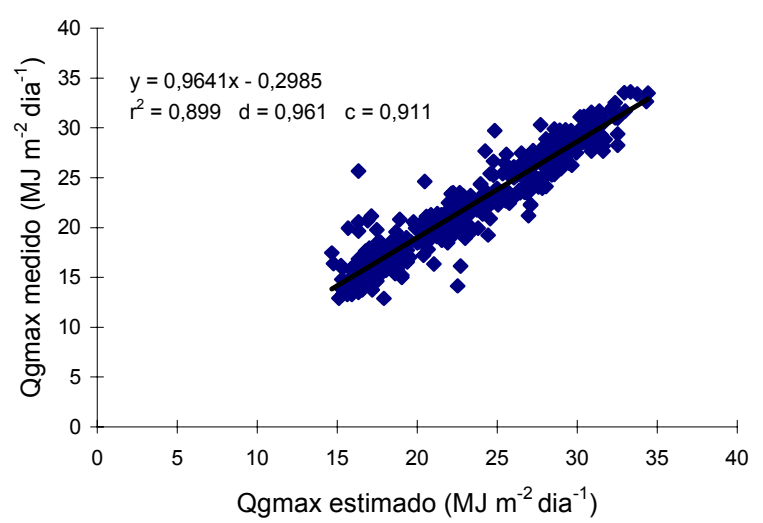

Figura 2 - Comparação entre dados medidos e estimados de potencial de energia solar, considerando uma série independente de dados para Ponta Grossa, PR. 
Ponta Grossa, PR 17/12/90

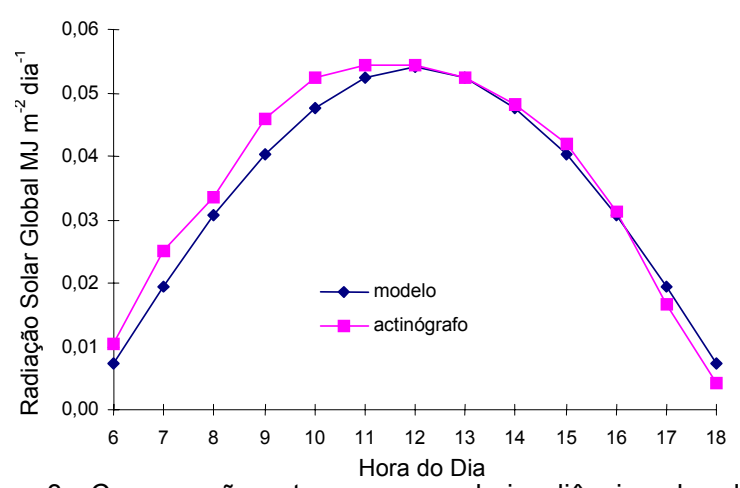

Ponta Grossa, PR 03/12/91

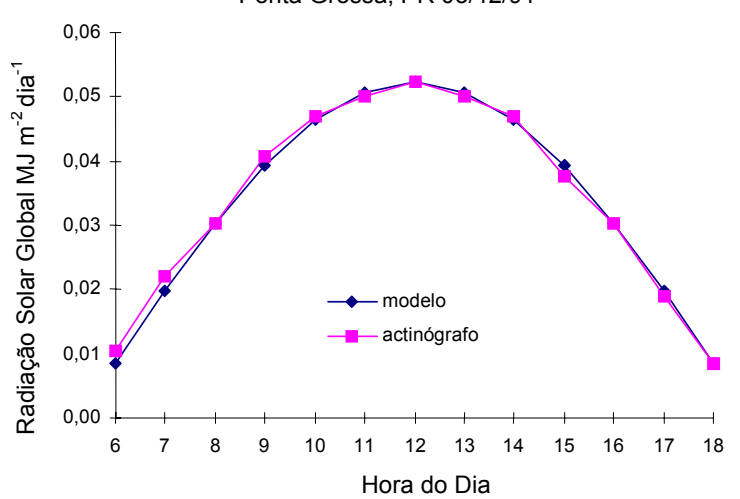

Figura 3 - Comparação entre as curvas de irradiância solar global ao longo do dia medida pelo actinógrafo e estimada pela metodologia proposta por Pereira \& Villa Nova (1998) para Ponta Grossa, PR.
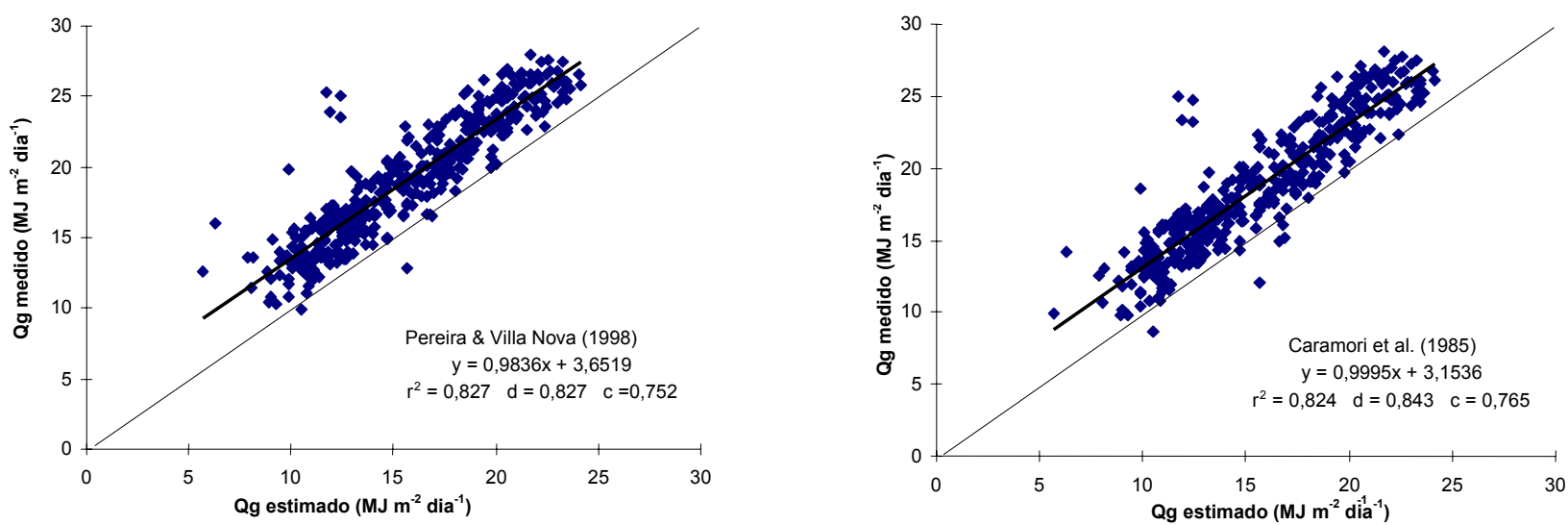

Figura 4 - Comparação entre a irradiância solar global medida pelo actinógrafo e a irradiância estimada a partir dos coeficientes a e b da equação de Angström propostos por Caramori et al. (1985) e pela metodologia em estudo para Ponta Grossa, PR.

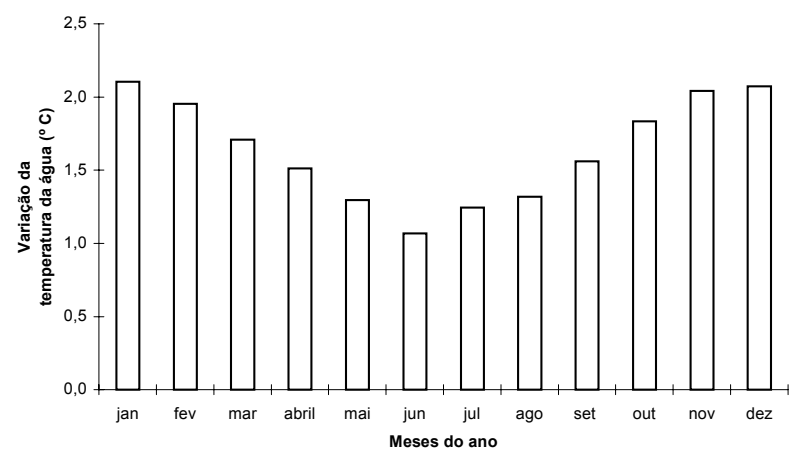

Figura 5 - Potencial de acréscimo de temperatura da água em coletor solar plano ao longo do ano, em função do potencial de energia solar disponível na superfície em Ponta Grossa, PR.

Neste estudo preliminar, são observados incrementos médios mensais de temperatura da água para cada metro quadrado de coletor, instalado na horizontal no município de Ponta Grossa, PR, por metro cúbico de água, considerando-se o rendimento médio dos coletores industriais de $30 \%$, bem como o período de 18 meses para obtenção dos valores de potencial de energia solar disponível (Figura 5). A metodologia desenvolvida provê, então, dados necessários para dimensionamento de coletores solares planos para fins de aquecimento de água (residencial, hospitalar, industrial etc.), permitindo a avaliação da máxima performance possível desses sistemas de captura de energia, ao longo do ano, na localidade estudada.

\section{CONCLUSÕES}

É possível estimar-se com boa precisão em Ponta Grossa, PR, o máximo "imput" de energia solar diário, através de apenas uma medida instantânea de intensidade radiométrica feita na passagem meridiana do sol.

A metodologia proposta mostrou-se eficiente para avaliar os parâmetros empíricos da regressão da equação de Angström com rapidez e simplicidade, permitindo a determinação da densidade de fluxo de radiação solar global diária no local em estudo.

\section{AGRADECIMENTOS}

À Divisão de Agrometeorologia, do Instituto Agronômico do Paraná - IAPAR/Londrina particularmente, ao pesquisador Dr. Paulo Henrique Caramori e ao meteorologista Edmirson Borrozzino, pela concessão dos dados radiométricos no período em estudo. Ao Conselho Nacional de Desenvolvimento Científico e Tecnológico - CNPq - pelo apoio financeiro para realização do presente trabalho. 


\section{REFERÊNCIAS BIBLIOGRÁFICAS}

AMPRATWUM, D.B.; DORVLO, A.S.S. Estimation of solar radiation from the number of sunshine hours. Applied Energy, v.63, p.161-167, 1999.

ASSIS, F.N.; STEINMETZ S.; MARTINS, S.R.; MENDES, M.E.G Aplicabilidade de modelos de estimativa da radiação solar global. Revista Brasileira de Agrometeorologia, v.6, p.91-97. 1998.

CAMARGO, A.P.; SENTELHAS, P.C. Avaliação de modelos para estimativa da evapotranspiração potencial mensal, em base diária para Campinas e Ribeirão Preto, SP. In: CONGRESSO BRASILEIRO DE AGROMETEOROLOGIA, 7., Campina Grande, 1995. Resumos. Campina Grande: Sociedade Brasileira de Agrometeorologia, 1995. p.415-417.

CARAMORI, P.H.; CORREA, A.R.; BORROZZINO, E. Estimativa da radiação solar global diária para Ponta Grossa, PR, a partir da insolação diária Poliagro, v.7, p.107-118, 1985.

ELAGIB, N.A.; MANSELL, M.G. New approaches for estimating global solar radiation across Sudan. Energy Conservation and Management, v.41, p.419-434, 2000.

PENMAN, H.L. Natural evaporation from open water, bare soil and grass. Proceedings of the Royal Society of London. Series A, v.193 p.120-145, 1948.

PRESCOTT, J.A. Evaporation from a water surface in relation to solar radiation. Transaction of the Royal Society and Science, v.64, p.114-125, 1940.

PEREIRA, A.B.; VILLA NOVA, N.A. Modelo de estimativa do potencial de energia solar diária disponível no município de Botucatu, SP, Brasil. Energia na Agricultura, v.13, p.38-50, 1998.

PEREIRA, A.B.; VILLA NOVA, N.A.; ESCOBEDO, J.F.; OLIVEIRA, A Evaluation of the solar energy potential at surface in São Paulo, SP, Brazil. Revista Brasileira de Agrometeorologia, v.6, p.99-104, 1998.
RAMACHANDA, T.V.; SUBRAMANIAN, D.K. Potential and prospects of solar energy in Uttara Kannada, District of Karnataka State, India. Energy Sources, v.19, p.945-988, 1997.

ROBINSON, N. The radiation emitted by the sun. In: ROBINSON, N. Solar radiation. Amsterdam: Elsevier, 1966. p.1-28.

SAHIN, A.D.; SEN, Z. Statistical analysis of the Angström formula coefficients and application for Turkey. Solar Energy, v.62, p.29-38, 1998.

SANTOS, R.; ANDRÉ, R.G.B.; VOLPE, C.A. Estimativa da radiação solar global em Jaboticabal, SP. Científica, v.11, p.31-39, 1983.

VILLA NOVA, N.A.; SALATI, E. Radiação solar no Brasil. In: SIMPÓSIO ANUAL DA ACADEMIA DE CIÊNCIAS DO ESTADO DE SÃO PAULO, 2. São Paulo, 1977. Anais. São Paulo: Academia de Ciências do Estado de São Paulo, 1977. p.27-61.

VILLA NOVA, N.A.; PEREIRA, A.B. Avaliação do potencial de energia solar no município de Piracicaba, SP, Brasil. In: CONGRESSO BRASILEIRO DE AGROMETEOROLOGIA, 10., Piracicaba, 1997. Anais. Piracicaba: Sociedade Brasileira de Agrometeorologia, 1997. p.413-415.

VRISMAN, A.L. Avaliação da energia solar máxima incidente na região de Ponta Grossa, PR. Ponta Grossa, 2000. 80p. Monografia (Agrometeorologia) - Universidade Estadual de Ponta Grossa.

WILLMOTT, C.J.; ACKLESON, S.G.; DAVIES, R.E.; FEDDEMA, J.J.; KLINK, K.M.; LEGATES, D.R.; O'DONNELL, J.; ROWE, C.M. Statistics for the evaluation and comparison of models. Journal of Geophysical Research, v.90, p.8995-9005, 1985.

Recebido em 05.01 .01 